\title{
Estimação de estados utilizando Observador Takagi-Sugeno e Filtro de Partículas - Uma aplicação para controle termoelétrico não linear
}

\author{
Letícia M. S. Vianna*, Márcio F. Braga**, \\ Mateus Giesbrecht*, Víctor C. S. Campos *** \\ * Faculdade de Engenharia Elétrica e de Computação, Universidade \\ Estadual de Campinas - UNICAMP, Campinas, SP, (e-mail: \\ leticiav@dsif.fee.unicamp.br,mateus@fee.unicamp.br) \\ ** Departamento de Engenharia Elétrica, Instituto de Ciências Exatas \\ e Aplicadas - ICEA, Universidade Federal de Ouro Preto - UFOP, \\ João Monlevade, MG (e-mail: mfbraga@ufop.edu.br) \\ *** Departamento de Engenharia Eletrônica, Escola de Engenharia, \\ Universidade Federal de Minas Gerais - UFMG, Belo Horizonte, MG \\ (e-mail:victor@cpdee.ufmg.br)
}

\begin{abstract}
The aim of this paper is to present and compare two state estimation methods, performed off-line, which are implemented in a thermoelectrically controlled chamber. The thermoelectrically controlled chamber consists of a chamber equipped with five digital temperature sensors scattered through the chamber, a cooling/heating actuator composed of Peltier modules, which are usually more ecofriendly than conventional cooling systems. The states of the plant consist in the temperatures measured by the five digital temperature sensors. In this paper, the methods chosen to estimate the states are the particle filter and a Takagi-Sugeno fuzzy observer designed with Linear Matrix Inequalities conditions. A comparison between the two state estimation approaches is performed and illustrated in the paper.

Resumo: O objetivo deste trabalho é apresentar e comparar dois métodos de estimação de estado, realizados off-line, que são aplicados em uma câmara termoeletricamente controlada, que consiste de uma câmara equipada com cinco sensores digitais de temperatura e um atuador de resfriamento/aquecimento composto de módulos Peltier. Os estados do sistema consistem nas temperaturas medidas pelos cinco sensores digitais de temperatura. Neste trabalho, os métodos escolhidos para estimar os estados foram o Filtro de Partículas e um Observador Fuzzy Takagi-Sugeno projetado com condições baseadas em desigualdades matriciais lineares. Uma comparação entre as duas abordagens de estimação de estado é realizada e ilustrada no artigo.

Keywords: State estimation, Takagi-Sugeno Observer, Particle Filter, Fuzzy, LMI Application. Palavras-chaves: Estimação de estados, Observador Takagi-Sugeno, Filtro de Partículas, Fuzzy, Aplicação de LMI.
\end{abstract}

\section{INTRODUÇÃO}

O problema de estimação de estados de sistemas com parâmetros incertos tem sido bastante estudado, visto que o problema permite além da estimação de estados a estimação de parâmetros não acessíveis a medições (Xie et al., 2014; Borges et al., 2008; Chong et al., 2015).

Para solucionar o problema de estimação de estados, elaboraram-se várias metodologias, como em Kalman (1960), no qual é proposto o Filtro de Kalman, método de filtragem e predição utilizando processos estocásticos

\footnotetext{
* Os autores agradecem ao apoio financeiro recebido da Coordenação de Aperfeiçoamento de Pessoal de Nível Superior - Brasil (CAPES) - Código de Financiamento 001, do Conselho Nacional de Desenvolvimento Científico e Tecnológico (CNPq) e da Universidade Federal de Ouro Preto (Número do Processo 23109.003268/2017-47).
}

gaussianos em sistemas dinâmicos lineares. Entretanto, métodos desenvolvidos para o tratamento de sistemas lineares não funcionam de maneira eficaz em sistemas não lineares. Assim, métodos para tratarem tais sistemas foram e são desenvolvidos, tal como o Filtro de Kalman Estendido (EKF), que teve uma de suas primeiras aplicações apresentada em Kappl (1971) e o Filtro de Partículas, apresentado em várias referências tais como Simon (2006).

Os filtros de Partículas são usualmente aplicados a problemas altamente não lineares que possuem estados/parâmetros desconhecidos do sistema. O filtro é um método estatístico baseado em probabilidade e no teorema de Bayes (Speekenbrink, 2016), sendo conhecido por diversos nomes, incluindo Filtro Sequencial de Monte Carlo (Wang et al., 2009). 
Algumas aplicações incluem o posicionamento de objetos rígidos e articulados (Hue et al., 2002; Ye and Liu, 2006) e a estimação de parâmetros de uma série temporal do Índice de Vegetação da Diferença Normalizada (NDVI, do inglês Normalized Difference Vegetation Index), a qual analisa a condição da vegetação natural ou agrícola nas imagens geradas por sensores remotos (Chakraborty et al., 2017).

Outra técnica de estimação de estados não linear faz uso do modelo fuzzy Takagi-Sugeno (TS), a qual consiste em descrever o sistema não linear como uma combinação convexa de modelos lineares (Takagi and Sugeno, 1985; Tanaka et al., 1998) por meio de regras se-então. Tais modelos são capazes de representar o comportamento do sistema em diferentes pontos do espaço de estados (Teixeira and Zak, 1999). A técnica é amplamente estudada devido à sua capacidade de aproximação universal, que lhe permite considerar as não linearidades de um sistema. Além disso, o fato de sua estrutura de modelo ser semelhante à de sistemas lineares incertos (com incertezas politópicas) permite generalizar o controle e a estimativa por meio do uso de Desigualdades Matriciais Lineares (LMIs, do inglês Linear Matrix Inequalities) (Boyd et al., 1994; Chadli and Karimi, 2013) para o contexto de sistemas não lineares (Guerra et al., 2012; Chadli and Karimi, 2012).

Neste trabalho, os algoritmos de filtragem de partículas e do observador de estados TS (projetado com condições recentes de LMI) são apresentados e aplicados off-line a uma câmara termoeletricamente controlada. Os resultados de ambas as técnicas são comparados e apresentados.

O trabalho é organizado da seguinte forma: na Seção 2 apresenta-se o observador de estados TS. Na Seção 3 apresenta-se o estimador de estados via Filtro de Partículas. Na Seção 4, o sistema termoelétrico não linear é apresentado. Na Seção 5, exibem-se os resultados das estimativas dos estados juntamente com a análise estatística do modelo. Por fim, na Seção 6, são apresentadas as considerações finais e as comparações entre ambos os métodos utilizados.

Notação. Ao longo deste artigo, as variáveis maiúsculas são matrizes, as variáveis em negrito e minúsculas são vetores coluna e as variáveis minúsculas são escalares. $A^{T}$ representa a transposta da matriz $A$ e $A^{-1}$ indica a inversa da matriz $A . P>0(P \geq 0)$ indica que a matriz $P$ é definida positiva (semidefinida). O símbolo $*$ representa os termos simétricos em uma matriz simétrica e o índice temporal de um sinal é representado pela letra $k$.

São consideradas as seguintes notações abreviadas ao longo do artigo

$$
\begin{aligned}
Q_{h} & =\sum_{i=1}^{r} h_{i}\left(\mathbf{x}_{k}\right) Q_{i}, \\
Q_{h^{-}} & =\sum_{i=1}^{r} h_{i}\left(\mathbf{x}_{k-1}\right) Q_{i}, \\
Q_{h h^{-}} & =\sum_{i=1}^{r} \sum_{j=1}^{r} h_{i}\left(\mathbf{x}_{k}\right) h_{j}\left(\mathbf{x}_{k-1}\right) Q_{i j} .
\end{aligned}
$$

\section{OBSERVADOR DE ESTADOS TAKAGI-SUGENO}

Neste trabalho, considera-se um modelo TS discreto dado por

$$
\begin{aligned}
\mathbf{x}_{k+1} & =A_{h} \mathbf{x}_{k}+B_{h} \mathbf{u}_{k}, \\
\mathbf{y}_{k} & =C \mathbf{x}_{k},
\end{aligned}
$$

e um Observador Fuzzy, baseado no estimador proposto em Guerra et al. (2012) com as variáveis premissas medidas dadas por

$$
\begin{aligned}
\hat{\mathbf{x}}_{k+1} & =A_{h} \hat{\mathbf{x}}_{k}+B_{h} \mathbf{u}_{k}+M_{h^{-}}^{-1} N_{h h^{-}}\left(\mathbf{y}_{k}-\hat{\mathbf{y}}_{k}\right), \\
\hat{\mathbf{y}}_{k} & =C \hat{\mathbf{x}}_{k},
\end{aligned}
$$

em que $M_{h^{-}}$e $N_{h h^{-}}$são os ganhos do observador.

O erro de estimação tem dinâmica dada, por

$$
\mathbf{e}_{k+1}=\left(A_{h}-M_{h^{-}}^{-1} N_{h h^{-}} C\right) \mathbf{e}_{k},
$$

em que $\mathbf{e}_{k}=\mathbf{x}_{k}-\hat{\mathbf{x}}_{k}$ é dado pela diferença entre o estado observado e o estado estimado.

A análise de estabilidade do erro da estimação pode ser realizada utilizando uma função de Lyapunov com pertinência atrasada, conforme apresentado em (Guerra et al., 2012),

$$
V_{k}=\mathbf{e}_{k} P_{h^{-}} \mathbf{e}_{k}
$$

se existirem matrizes $P_{i}$ e $M_{i}$, com $i \in 1,2, \ldots, r$, e $N_{i j}$, com $i, j \in 1,2, \ldots, r$, em que $r$ é o número de regras seentão, tem-se

$$
\left[\begin{array}{cc}
-P_{j} & * \\
M_{j} A_{i}+N_{i j} C & P_{i}-G_{j}^{T}-G_{j}
\end{array}\right]<0, \quad \forall i, j .
$$

Se as condições forem satisfeitas, tem-se que o sistema é assintoticamente estável.

É importante considerar não apenas a estabilidade, mas também outros índices de desempenho do sistema, tais como a velocidade de resposta, restrições de entrada e saída. Em razão disso, o estimador Fuzzy com taxa de decaimento foi utilizado, o qual relaciona a velocidade de resposta com uma taxa de decaimento exponencial. Para garantir uma taxa de decaimento, é necessário uma função de Lyapunov para satisfazer a condição (Tanaka et al., 1998)

$$
V_{k+1}<\alpha^{2} V_{k}
$$

em que $\alpha \in(0,1)$ é a taxa de decaimento. Portanto, a condição LMI (10) é alterada para

$$
\left[\begin{array}{cc}
-\alpha^{2} P_{j} & * \\
M_{j} A_{i}+N_{i j} C & P_{i}-G_{j}^{T}-G_{j}
\end{array}\right]<0 .
$$

Para realizar tal estimação é feita uma busca unidimensional, encontrando o menor valor para $\alpha$ que solucione a LMI (12), resolvendo assim o problema de minimização (Tanaka et al., 1998).

\section{FILTRO DE PARTÍCULAS}

O Filtro de Partículas é um estimador bayesiano baseado em probabilidade (Ching et al., 2006). Este algoritmo pertence à classe de filtros estocásticos, sendo portanto uma técnica de Monte Carlo para a solução de problemas de estimação de estados e de parâmetros em sistemas não lineares e não gaussianos (Simon, 2006). 
Dado o sistema

$$
\begin{aligned}
\mathbf{x}_{k+1} & =\mathbf{f}_{k}\left(\mathbf{x}_{k}, \mathbf{u}_{k}, \boldsymbol{\omega}_{k}\right), \\
\mathbf{y}_{k} & =\mathbf{h}_{k}\left(\mathbf{x}_{k}, \boldsymbol{\nu}_{k}\right),
\end{aligned}
$$

em que $\mathbf{x} \in \mathbb{R}^{n}, \mathbf{y} \in \mathbb{R}^{l}, \mathbf{u} \in \mathbb{R}^{m}$ são vetores de estados, observações e entradas, respectivamente. Os vetores $\boldsymbol{\omega}_{k} \mathrm{e}$ $\boldsymbol{\nu}_{k}$ são vetores de ruído de processo e de ruído de observação, respectivamente. Ambos os ruídos são considerados mutuamente independentes e com função de densidade de probabilidade (pdf, do inglês probability density function) conhecidas. $\mathbf{f}_{k}(\cdot)$ e $\mathbf{h}_{k}(\cdot)$ são funções não lineares variantes no tempo que representam o sistema.

O processo de filtragem é descrito como segue: inicialmente um conjunto de $N$ de vetores de estados, chamados de partículas, é gerado baseado na pdf do estado inicial $p\left(\mathbf{x}_{0}\right)$, assumindo que seja conhecida. O número $N$ de partículas é escolhido levando em consideração o custo computacional e a qualidade da estimação (Wang et al., 2009).

Posteriormente, as partículas são propagadas/atualizadas no espaço de estados por (13). Esse passo representa a distribuição a priori das partículas no intervalo que está sendo evoluído.

Considera-se que a distribuição da observação y é dada pela distribuição gaussiana

$$
p\left(\mathbf{y}_{k} \mid \mathbf{x}_{k}, \boldsymbol{\nu}_{k}\right) \sim \mathcal{N}(\hat{\mathbf{y}}, R)
$$

em que $R$ é a matriz de covariância do ruído de observação, $\boldsymbol{\nu}_{k} \sim \mathcal{N}(0, R)$, e $\hat{\mathbf{y}}=C \mathbf{x}_{k}$ o valor esperado da observação. Assim, são calculados pesos para cada uma das partículas, que são medidas da probabilidades da observação da saída para o valor dos estados a priori, sendo calculado por

$$
q_{p_{k}}=\exp \left(\frac{-\left[\mathbf{y}_{k}^{*}-\hat{\mathbf{y}}_{p_{k}}\right]^{T} R^{-1}\left[\mathbf{y}_{k}^{*}-\hat{\mathbf{y}}_{p_{k}}\right]}{2}\right)
$$

$\operatorname{com} p=1, \ldots, N$ e $\mathbf{y}^{*}$ a observação real (Simon, 2006).

Após realizado o cálculo, os pesos são normalizados por

$$
q_{p}=\frac{q_{p}}{\sum_{j=1}^{N} q_{j}},
$$

e, assim, a distribuição a posteriori das partículas é gerada pela reamostragem das partículas de acordo com os pesos. Note que os pesos representam a probabilidade da partícula ser selecionada para fazer parte da distribuição a posteriori.

A partir do conjunto de partículas a posteriori, calculase alguma medida estatística, média ou covariância, para estimar os estados.

\section{CÂMARA TERMOELETRICAMENTE CONTROLADA}

Ambos os estimadores foram aplicados em uma Câmara Termoeletricamente Controlada, apresentada na Figura 1, a qual possui um total de cinco sensores digitais de temperatura do tipo DS18B20, que foram configurados para ter uma precisão de $0,0625^{\circ} \mathrm{C}$. Dentre os cinco sensores, três foram distribuídos no interior da câmara e dois ao seu redor como apresentado na Figura 2, onde também é apresentada a localização dos módulos Peltier. Maiores informações sobre a parte experimental podem ser encontradas em Pereira et al. (2018).

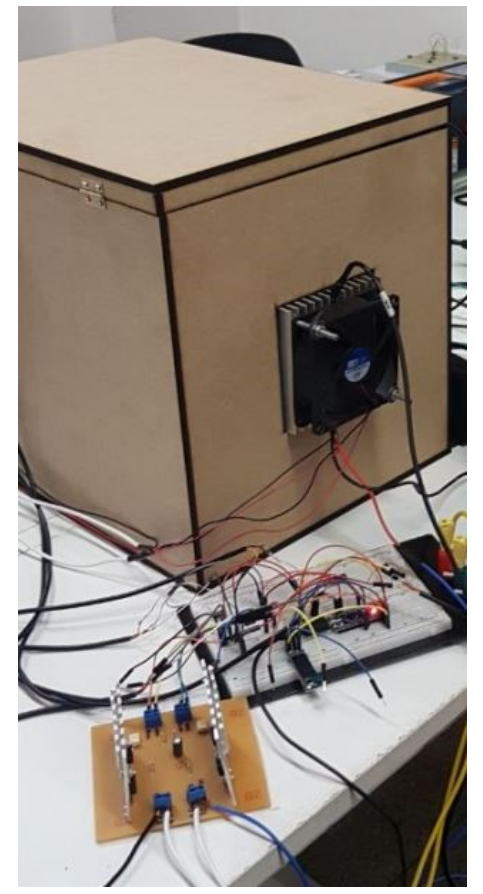

Figura 1. Câmara Termoeletricamente Controlada.

Os módulos Peltier (TEC 12715) que são acoplados a dissipadores de calor, responsáveis por aumentar a área superficial e a taxa de transferência de calor e coolers que auxiliam na distribuição homogênea do calor no interior da câmara.

A temperatura da câmara pode variar entre 16 e 50 graus Celsius e pode ser descrita por um modelo Fuzzy TS discreto com 5 estados e $r=13$ regras se-então. É importante notar que cada sensor de temperatura descreve um estado.

Para a obtenção do modelo da câmara, foram empregadas técnicas de identificação de sistemas usando uma abordagem de erro quadrático médio (Takagi and Sugeno, 1985). A descrição do procedimento de identificação da câmara pode ser encontrada em Pereira et al. (2018).
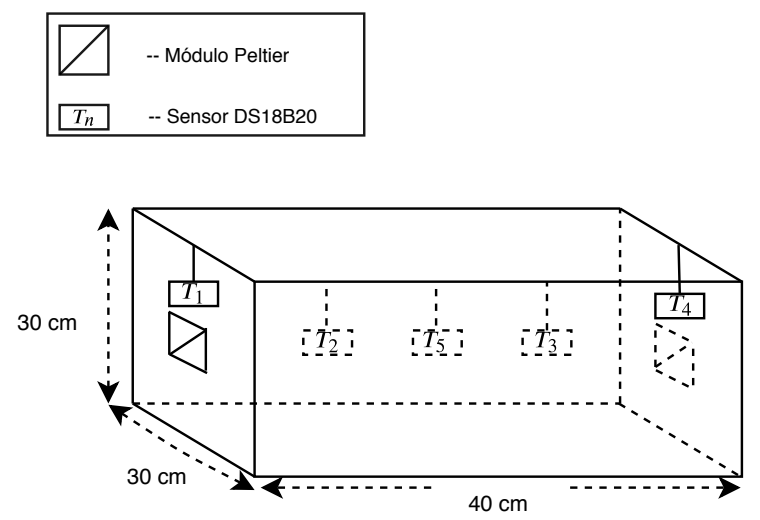

Figura 2. Localizações dos sensores de temperatura e módulos Peltier na Câmara.

O modelo considera que a única variável premissa é o quinto estado, $\mathbf{x}_{5}$, que representa a temperatura no centro da câmara. 
As funções de pertinência, $h_{i}$, foram fixadas antes do processo de identificação, sendo dadas por

$$
\begin{aligned}
& \mu_{i}\left(\mathbf{x}_{5}\right)=\exp \left(\frac{-\left(\mathbf{x}_{5}-\theta_{i}\right)^{2}}{2 \sigma_{i}^{2}}\right), \\
& h_{i}\left(\mathbf{x}_{5}\right)=\frac{\mu_{i}\left(\mathbf{x}_{5}\right)}{\sum_{\ell=1}^{r} \mu_{\ell}\left(\mathbf{x}_{5}\right)},
\end{aligned}
$$

em que $i=1, \ldots, r$; com $\sigma_{i}=0,7111$ para todo $i$ e $\theta_{i}$ são valores igualmente espaçados entre 16,6 e 42,2.

Assim, tem-se a representação do sistema termoelétrico no espaço de estados

$$
\begin{aligned}
\mathbf{x}_{k+1} & =\mathbf{f}_{k}\left(\mathbf{x}_{k}, \mathbf{u}_{k}\right), \\
\mathbf{y}_{k} & =C \mathbf{x}_{k},
\end{aligned}
$$

em que o vetor de estados é dado por

$$
\begin{aligned}
\mathbf{x}_{k} & =\left[\begin{array}{lllll}
\mathbf{x}_{1_{k}} & \mathbf{x}_{2_{k}} & \mathbf{x}_{3_{k}} & \mathbf{x}_{4_{k}} & \mathbf{x}_{5_{k}}
\end{array}\right]^{\mathrm{T}}, \\
& =\left[\begin{array}{lllll}
\mathbf{T}_{1_{k}} & \mathbf{T}_{2_{k}} & \mathbf{T}_{3_{k}} & \mathbf{T}_{4_{k}} & \mathbf{T}_{5_{k}}
\end{array}\right]^{\mathrm{T}},
\end{aligned}
$$

$$
\mathbf{f}_{k}\left(\mathbf{x}_{k}, \mathbf{u}_{k}\right)=\sum_{i} h_{i}\left[A_{i} \mathbf{x}_{k}+B_{i} \mathbf{u}_{k}\right]
$$

é a função não linear de evolução de estados, com

$$
\mathbf{u}_{k}=\left[\mathbf{V}_{k}\right]^{T}, \quad \mathbf{y}_{k}=\left[\begin{array}{ll}
\mathbf{T}_{1_{k}} & \mathbf{T}_{5_{k}}
\end{array}\right]^{T}
$$

$$
C=\left[\begin{array}{lllll}
1 & 0 & 0 & 0 & 0 \\
0 & 0 & 0 & 0 & 1
\end{array}\right]
$$

a matriz de saída do sistema, $\mathbf{T}(\cdot)$ são as temperaturas dos sensores e $\mathbf{V}$ a tensão aplicada.

As matrizes $A_{i}$ e $B_{i}$ do sistema foram retiradas de Pereira et al. (2018).

\section{RESULTADOS}

A fim de testar as estratégias consideradas neste artigo, apenas o primeiro estado $\mathbf{x}_{1}$ e o quinto estado $\mathbf{x}_{5}$ foram utilizados no algoritmo de estimação de estados. Os demais estados também foram medidos, mas não foram considerados no algoritmo de estimação de estados, para se ter uma comparação entre os valores reais e os estimados pelos algoritmos propostos. Os algoritmos foram implementados no software Matlab R2016a em um computador pessoal com Intel@ Core $^{\mathrm{TM}}$ i3-4010Ux64 CPU @1.70GHz, 4.00 GB RAM, com sistema operacional Microsoft(c) Windows 10.

Os resultados obtidos para a estimação das temperaturas por meio do Filtro de Partículas foram analisados e comparados com os resultados encontrados por meio do Observador Fuzzy. Na Tabela 1 são apresentados os Erros Quadráticos Médios (EQM) calculados para os sinais das temperaturas estimadas com a execução do Filtro de Partículas (FP) com diferentes números $N$ de partículas. $\mathrm{O}$ sobrescrito $\sim$ representa o EQM de estimação do estado. Da tabela pode-se observar a relação direta entre o aumento no número das partículas utilizadas com o aumento da exatidão mas também um aumento no tempo de execução do algoritmo. Na Tabela 2 são apresentados os EQMs calculados para o sinal das temperaturas estimadas com a execução do Observador Fuzzy.

Nas figuras 3, 4, 5, 6 e 7 são apresentadas as estimações dos estados por meio do Filtro de Partículas, com 1000 partículas, e por meio do Observador Fuzzy. No Filtro

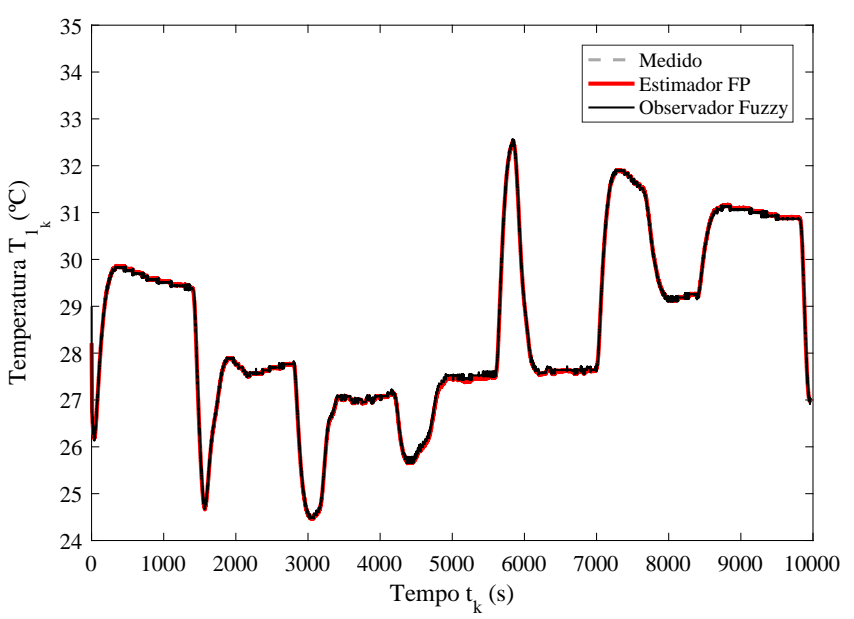

Figura 3. Estimação do estado $\mathbf{T}_{1_{k}}$. A curva pontilhada, em cinza, representa o estado medido, a curva contínua, em vermelho, o estado estimado via Filtro de Partículas e a curva contínua, em preto, o estado estimado via Observador Fuzzy.

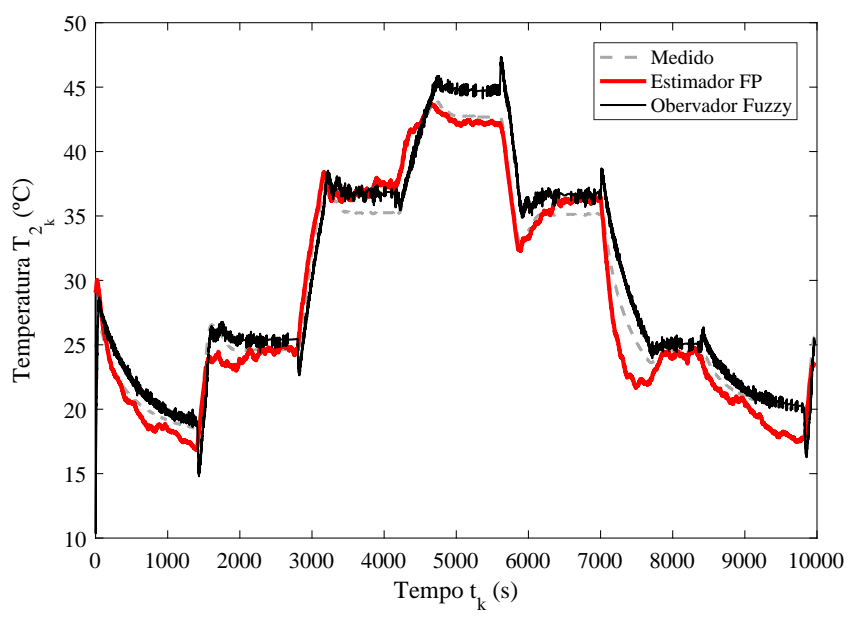

Figura 4. Estimação do estado $\mathbf{T}_{2_{k}}$. A curva pontilhada, em cinza, representa o estado medido, a curva contínua, em vermelho, o estado estimado via Filtro de Partículas e a curva contínua, em preto, o estado estimado via Observador Fuzzy.

de Partículas, foram adicionados ruídos de processo do

Tabela 1. Erro Quadrático Médio para diferentes números de partículas em ${ }^{\circ} \mathrm{C}$.

\begin{tabular}{ccccccc}
\hline $\mathrm{N}$ & $\tilde{\mathbf{T}}_{1}$ & $\tilde{\mathbf{T}}_{2}$ & $\tilde{\mathbf{T}}_{3}$ & $\tilde{\mathbf{T}}_{4}$ & $\tilde{\mathbf{T}}_{5}$ & Tempo execução(s) \\
\hline 50 & 0,002 & 5,006 & 1,955 & 1,068 & 0,003 & 74,289 \\
100 & 0,002 & 3,563 & 1,192 & 1,049 & 0,001 & 150,130 \\
500 & 0,000 & 2,056 & 0,989 & 0,740 & 0,001 & 729,428 \\
1000 & 0,000 & 1,957 & 0,783 & 0,653 & 0,000 & 1708,0 \\
\hline
\end{tabular}

Tabela 2. Erro Quadrático Médio para Observador $\mathrm{TS}$ em ${ }^{\circ} \mathrm{C}$.

\begin{tabular}{cccccc}
\hline$\tilde{\mathbf{T}}_{1}$ & $\tilde{\mathbf{T}}_{2}$ & $\tilde{\mathbf{T}}_{3}$ & $\tilde{\mathbf{T}}_{4}$ & $\tilde{\mathbf{T}}_{5}$ & Tempo execução(s) \\
\hline 0,038 & 1,693 & 1,758 & 0,692 & 0,048 & 208,473 \\
\hline
\end{tabular}




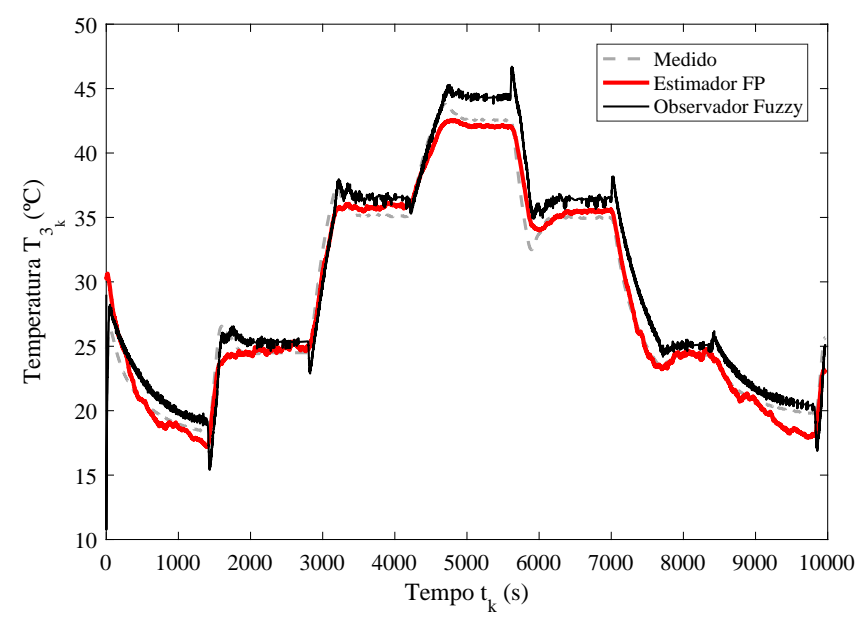

Figura 5. Estimação do estado $\mathbf{T}_{3_{k}}$. A curva pontilhada, em cinza, representa o estado medido, a curva contínua, em vermelho, o estado estimado via Filtro de Partículas e a curva contínua, em preto, o estado estimado via Observador Fuzzy.

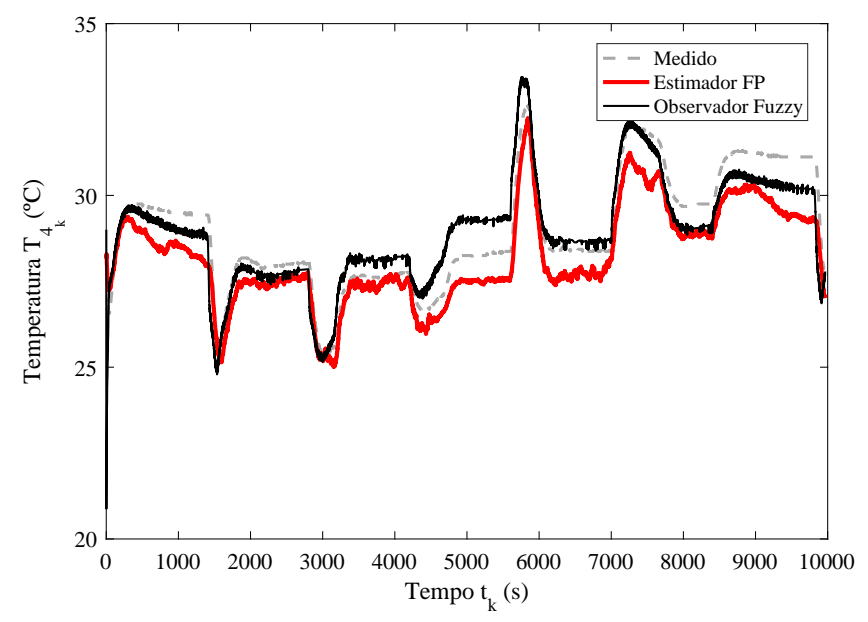

Figura 6. Estimação do estado $\mathbf{T}_{4_{k}}$. A curva pontilhada, em cinza, representa o estado medido, a curva contínua, em vermelho, o estado estimado via Filtro de Partículas e a curva contínua, em preto, o estado estimado via Observador Fuzzy.

tipo branco e gaussiano com o objetivo de representar as incertezas à equação de evolução de estados, $\boldsymbol{\omega}_{k} \sim$ $\mathcal{N}(0 ; 0,0156)$.

Quanto à estimação por meio do Observador Fuzzy, a LMI foi solucionada utilizando os pacotes do software Matlab: SeDuMi (Sturm, 1999) e Yalmip (Lofberg, 2004). Sendo o menor valor de $\alpha=0,9777$ encontrado por meio de uma busca unidimensional realizada off-line.

Os estados $\mathbf{T}_{1}$ e $\mathbf{T}_{4}$, nas figuras 3 e 6 , apresentam dinâmicas diferentes dos demais por estarem localizados na parte externa na câmara.

Para estados não medidos, ou seja, $\mathbf{T}_{2}, \mathbf{T}_{3}$ e $\mathbf{T}_{4}$ o TS teve um erro menor que o Filtro de Partículas, entretanto para estados medidos o Filtro de Partículas se comportou melhor. Mesmo com 1000 partículas o filtro não obteve

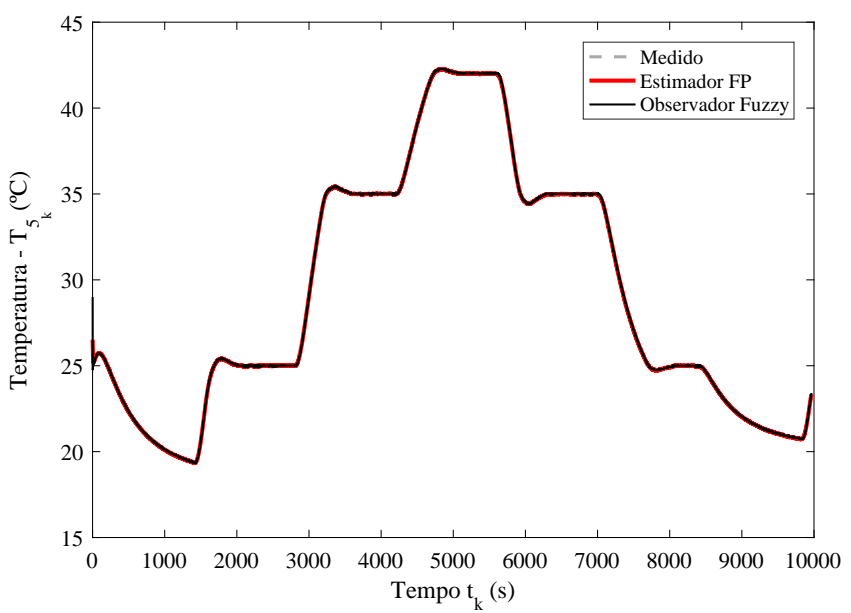

Figura 7. Estimação do estado $\mathbf{T}_{5_{k}}$. A curva pontilhada, em cinza, representa o estado medido, a curva contínua, em vermelho, o estado estimado via Filtro de Partículas e a curva contínua, em preto, o estado estimado via Observador Fuzzy.

uma precisão tão boa quanto a do TS para estados não observados, embora o tempo tenha sido muito maior que o TS. Vale ressaltar que para até 50 partículas o filtro é mais rápido que o TS, entretanto os resultados não foram tão bons. Por fim, o erro para estados medidos é sempre menor que para estados não medidos.

\section{CONCLUSÃO}

Neste trabalho foi proposta a aplicação off-line de duas diferentes estratégias usando dados de uma sistema real. Os métodos propostos, Filtro de Partículas e Observador Fuzzy, foram aplicados em uma Câmara Termoeletricamente Controlada que consiste em uma câmara equipada com cinco sensores digitas de temperatura fixados em sua superfície, representando os estados da planta, e um atuador de resfriamento/aquecimento composto por módulos Peltier. Os resultados obtidos apresentam boas estimativas para ambos os métodos, quando analisados os erros quadráticos médios dos estados estimados em relação aos dados medidos. Ao comparar os resultados obtidos pelas duas abordagens, o Observador Fuzzy obteve bons resultados em um menor intervalo de tempo quando comparado com a melhor estimativa encontrada pelo Filtro de Partículas, com $N=1000$ partículas, para os estados não medidos.

As estimações foram realizadas offline, entretanto para trabalhos futuros pretende-se verificar o que os diferentes estimadores causam sobre a malha fechada. Além de empregar outras técnicas de estimação, tal como o Filtro de Kalman Estendido e comparar os resultados.

\section{REFERÊNCIAS}

Borges, R.A., Montagner, V.F., Oliveira, R.C., Peres, P.L.D., and Bliman, P.A. (2008). Parameter-dependent $\mathcal{H}_{2}$ and $\mathcal{H}_{\infty}$ filter design for linear systems with arbitrarily time-varying parameters in polytopic domains. Signal Processing, 88(7), 1801-1816.

Boyd, S., El Ghaoui, L., Feron, E., and Balakrishnan, V. (1994). Linear Matrix Inequalities in System and 
Control Theory. SIAM Studies in Applied Mathematics, Philadelphia, PA.

Chadli, M. and Karimi, H.R. (2012). On the robust design of unknown inputs Takagi-Sugeno observer. In Decision and Control (CDC), 2012 IEEE 51st Annual Conference on, 4770-4773. IEEE.

Chadli, M. and Karimi, H.R. (2013). Robust observer design for unknown inputs Takagi-Sugeno models. IEEE Transactions on Fuzzy Systems, 21(1), 158-164.

Chakraborty, S., Banerjee, A., Gupta, S., PapandreouSuppappola, A., and Christensen, P. (2017). Estimation of dynamic parameters of MODIS NDVI time series nonlinear model using particle filtering. In 2017 IEEE International Geoscience and Remote Sensing Symposium (IGARSS), 1091-1094. IEEE.

Ching, J., Beck, J.L., and Porter, K.A. (2006). Bayesian state and parameter estimation of uncertain dynamical systems. Probabilistic engineering mechanics, 21(1), 8196.

Chong, M.S., Nešić, D., Postoyan, R., and Kuhlmann, L. (2015). Parameter and state estimation of nonlinear systems using a multi-observer under the supervisory framework. IEEE Transactions on Automatic Control, 60(9), 2336-2349.

Guerra, T.M., Kerkeni, H., Lauber, J., and Vermeiren, L. (2012). An efficient Lyapunov function for discrete TS models: observer design. IEEE Transactions on Fuzzy Systems, 20(1), 187-192.

Hue, C., Le Cadre, J.P., and Pérez, P. (2002). Tracking multiple objects with particle filtering. IEEE transactions on aerospace and electronic systems, 38(3), 791-812.

Kalman, R.E. (1960). A new approach to linear filtering and prediction problems. Transactions of the ASMEJournal of Basic Engineering.

Kappl, J. (1971). Nonlinear estimation via Kalman filtering. IEEE Transactions on Aerospace and Electronic Systems, (1), 79-84.

Lofberg, J. (2004). YALMIP: A toolbox for modeling and optimization in MATLAB. In Computer Aided Control Systems Design, 2004 IEEE International Symposium on, 284-289. IEEE.

Pereira, A.M.F., Vianna, L.M.S., Keles, N.A., and da Silva Campos, V.C. (2018). Tensor product model transformation simplification of Takagi-Sugeno control and estimation laws-an application to a thermoelectric controlled chamber. Acta Polytechnica Hungarica, $15(3)$.

Simon, D. (2006). Optimal state estimation: Kalman, $\mathcal{H}_{\infty}$, and nonlinear approaches. John Wiley \& Sons.

Speekenbrink, M. (2016). A tutorial on particle filters. Journal of Mathematical Psychology, 73, 140-152.

Sturm, J.F. (1999). Using SeDuMi 1.02, a MATLAB toolbox for optimization over symmetric cones. Optimization methods and software, 11(1-4), 625-653.

Takagi, T. and Sugeno, M. (1985). Fuzzy identification of systems and its application to modeling and control. IEEE Transactions on Systems, Man, and Cybernetics, SMC-15(1), 116-132.

Tanaka, K., Ikeda, T., and Wang, H.O. (1998). Fuzzy regulators and fuzzy observers: relaxed stability conditions and LMI-based designs. IEEE Transactions on fuzzy systems, 6(2), 250-265.
Teixeira, M.C. and Zak, S.H. (1999). Stabilizing controller design for uncertain nonlinear systems using fuzzy models. IEEE Transactions on Fuzzy systems, 7(2), 133142.

Wang, D., Zhao, J., and Wang, W. (2009). Particle filter based robust mobile robot localization. In Mechatronics and Automation, 2009. ICMA 2009. International Conference on, 817-821. IEEE.

Xie, X.P., Yang, D.S., and Zhu, X.L. (2014). Relaxed observer design of discrete-time $\mathrm{T}-\mathrm{S}$ fuzzy systems via a novel multi-instant fuzzy observer. Signal Processing, 102, 296-303.

Ye, Z. and Liu, Z.Q. (2006). Tracking human hand motion using genetic particle filter. In Systems, Man and Cybernetics, 2006. SMC'06. IEEE International Conference on, volume 6, 4942-4947. IEEE. 